\title{
Computation of rational Szegő-Lobatto quadrature formulas
}

\author{
Adhemar Bultheel ${ }^{\mathrm{a}, 1,2, *}$, Pablo González-Vera ${ }^{\mathrm{b}, 1}$, Erik Hendriksen ${ }^{\mathrm{c}}$, Olav Njåstad $^{\mathrm{d}}$ \\ ${ }^{a}$ Dept. Computer Sc., K.U.Leuven, Celestijnenlaan 200A, 3001 Heverlee, Belgium \\ ${ }^{b}$ Dept. Mathematical Analysis, University of La Laguna, Tenerife, Spain \\ ${ }^{c}$ Beerstratenlaan 23, 2421GN Nieuwkoop, The Netherlands \\ ${ }^{d}$ Dept. of Mathematical Sciences, Norwegian Univ. of Science and Technology, Trondheim, Norway
}

\begin{abstract}
Szegô quadrature formulas are analogs of Gauss quadrature rules when dealing with the approximate integration of periodic functions, since they exactly integrate trigonometric polynomials of as high degree as possible, or more generally Laurent polynomials which can be viewed as rational functions with poles at the origin and infinity. When more general rational functions with prescribed poles on the extended complex plane not on the unit circle are considered to be exactly integrated, the so called "Rational Szegő Quadrature Formulas" appear. In this paper, some computational aspects concerning these quadratures are analyzed when one or two nodes are previously fixed on the unit circle.
\end{abstract}

Keywords: rational Szegó quadrature, rational Szegő-Radau quadrature, rational Szegó-Lobatto quadrature, maximal domain of validity,

2000 MSC: 42C05 41A55

\section{Introduction}

In this paper we shall be concerned with the computation of the nodes $\left\{\theta_{k n}\right\}_{k=1}^{n}$ and the corresponding weights $\left\{\lambda_{k n}\right\}_{k=1}^{n}$ of the quadrature formula $J_{n}(F)$ to approximate the integral $J_{\Omega}(f)$ where

$$
J_{n}(F)=\sum_{k=1}^{n} \lambda_{k n} f\left(\theta_{k n}\right) \quad \text { and } \quad J_{\Omega}(F)=\int_{-\pi}^{\pi} F(\theta) \Omega(\theta) \mathrm{d} \theta .
$$

It is assumed that $F$ and $\Omega$ are $2 \pi$-periodic functions. Hence, to approximate this integral, we propose a quadrature formula of the form $\sum_{k=1}^{n} \lambda_{k n} F\left(\theta_{k n}\right)$ with $\theta_{k n} \in[-\pi, \pi), k=1, \ldots, n$. Here we shall moreover assume that the nodes do not coincide, so that we have indeed an $n$-point formula. For theoretical convergence as well as for for numerical stability, it is necessary that the weights $\lambda_{k n}$ be positive, which is another condition we shall impose on our quadrature formula. Finally, note that for $F$ sufficiently smooth, we get a good approximation to $F(\theta)$ by truncating its trigonometric Fourier series expansion. Therefore one usually attempts to make the approximation of the integral "as good as possible" by requiring that the quadrature formula is exact when $F$ is replaced by any trigonometric polynomial of degree $N$ or lower, with $N$ as large as possible. The same ideas and conditions are used in Gaussian quadrature formulas. The only difference is that there the integrand need not be periodic, but smooth enough to be well approximated by a polynomial, so that there one requires that the quadrature formula is exact for all polynomials of degree $N$ or lower with $N$ as large as possible. For Gaussian quadrature formulas, it is known that the maximal degree of

\footnotetext{
${ }^{*}$ Corresponding author

Email addresses: adhemar.bultheel@cs.kuleuven.be (Adhemar Bultheel), pglez@ull.es (Pablo González-Vera), erik.hendriksen@planet.nl (Erik Hendriksen), njastad@math.ntnu.no (Olav Njåstad)

${ }^{1}$ This work is partially supported by the Direción Direción General de Programas y Transferencia de Conocimiento, Ministerio de Ciencia e Innovación of Spain under grant MTM2008-06671.

${ }^{2}$ The work of this author is partially supported by the Fund for Scientific Research (FWO), projects "RAM: Rational modelling: optimal conditioning and stable algorithms", grant \#G.0423.05 and the Belgian Network DYSCO (Dynamical Systems, Control, and Optimization), funded by the Interuniversity Attraction Poles Programme, initiated by the Belgian State, Science Policy Office. The scientific responsibility rests with the author.
} 
accuracy $N$ is $2 n-1$ for an $n$-point interpolatory quadrature formula, obtained as the integral of the polynomial of degree $n$ interpolating $F$ in the nodes $\theta_{k n}$ which are the $n$ zeros of the polynomial of degree $n$, orthogonal on $[-\pi, \pi]$ with respect to the weight $\Omega$.

At this point, it is convenient to translate this setting to the unit circle. Given the fact that a function defined on the unit circle $\mathbb{T}=\{z \in \mathbb{C}:|z|=1\}$, whether it is real or complex, is always $2 \pi$-periodic. Hence the whole setting that was just explained can be reformulated as follows. Find a quadrature formula $I_{n}(f)$, approximating $I_{\omega}(f)$ where

$$
I_{n}(f)=\sum_{k=1}^{n} \lambda_{k n} f\left(z_{k n}\right) \quad \text { and } \quad I_{\omega}(f)=\int_{\mathbb{T}} f(z) \mathrm{d} \mu(z) \equiv \int_{-\pi}^{\pi} f\left(\mathrm{e}^{\mathrm{i} \theta}\right) \omega(\theta) \mathrm{d} \theta .
$$

(We assume $\omega(\theta)>0$ a.e. on $[-\pi, \pi]$.) The weights $\lambda_{k n}$ should all be positive, the nodes $z_{k n}$ should all be on $\mathbb{T}$, and the integral should be exact for all Laurent polynomials in $\Lambda_{-p, q}=\operatorname{span}\left\{z^{j}:-p \leq j \leq q\right\}$ with $N=p+q$ as large as possible. These analogs of the Gaussian quadrature formulas are called Szegó quadrature formulas. The main difference with Gaussian quadrature formulas is that the zeros of orthogonal polynomials on $\mathbb{T}$ are in the open unit disk $\mathbb{D}=\{z \in \mathbb{C}:|z|<1\}$, and not on $\mathbb{T}$. Instead the nodes should be chosen as the zeros of the so called para-orthogonal polynomials. These can be constructed as follows: Suppose $\rho_{n}(z)$ is the $n$th orthogonal polynomial and $\rho_{n}^{*}(z)=z^{n} \overline{\rho_{n}(1 / \bar{z})}$, then $Q_{n}(z)=\rho_{n}(z)+\tau \rho_{n}^{*}(z)$ is para-orthogonal, which means that it is orthogonal to $\Lambda_{1, n-1}$. Moreover, when $\tau \in \mathbb{T}$, then all its zeros are simple and on $\mathbb{T}$. If we use these as the nodes of an $n$-point Szegó quadrature formula, then it will be exact in $\Lambda_{-(n-1), n-1}$ and it will have positive weights $[1,15,19]$. It is possible to consider a slightly more general situation in which it is required that the quadrature formulas are exact in a space $\Lambda_{-p(n), q(n)}$ with $N=p(n)+q(n)$ as large as possible. However, in order not to obscure our point in this paper, we do not go into details here and invite the reader to consult the literature, e.g., [8].

Now, let us go one step further. We continue working in the unit circle setting, but it is easy to translate this to a setting of $2 \pi$-periodic functions. Assume that $f$ is much better approximated by rational functions, instead of Laurent polynomials. Hence for a given sequence $\left\{\alpha_{k}: k=1,2, \ldots\right\} \subset \mathbb{D}$ (we assume $\alpha_{0}=0$ ) we consider the space

$$
\mathscr{L}_{n}=\operatorname{span}\left\{\frac{P_{n}}{\pi_{n}}: P_{n} \in \Lambda_{0, n}, \pi_{n}(z)=\prod_{k=1}^{n}\left(1-\bar{\alpha}_{k} z\right)\right\}=\operatorname{span}\left\{B_{k}\right\}_{k=0}^{n}
$$

where the Blaschke products $B_{k}$ are defined by $B_{0}=1$ and for $k=1,2, \ldots$

$$
B_{k}=\zeta_{k} B_{k-1}, \quad \zeta_{k}(z)=\frac{z-\alpha_{k}}{1-\bar{\alpha}_{k} z}
$$

The space of polynomials forms a special case obtained when we set all $\alpha_{k}=0$. Thus for $p$ and $q$ nonnegative integers, the rational (i.e. multipoint) analog of the space of Laurent polynomials is then

$$
\mathscr{R}_{-p, q}=\mathscr{L}_{p *}+\mathscr{L}_{q}, \text { where } \mathscr{L}_{p *}=\left\{f_{*}: f \in \mathscr{L}_{p}\right\}, \text { and } f_{*}(z)=\overline{f(1 / \bar{z})}
$$

Note that the functions of $\mathscr{L}_{n}$ are analytic in $\mathbb{D}$ since all its poles are in the set $\left\{1 / \bar{\alpha}_{k}\right\}_{k=1}^{n}$. Hence the functions in $\mathscr{L}_{n *}$ are analytic in $\mathbb{E}=\{z \in \mathbb{C}:|z|>1\}$ because they have all their poles in $\left\{\alpha_{k}\right\}_{k=1}^{n}$, so that the functions in $\mathscr{R}_{-p, q}$ have their poles in $\left\{\alpha_{k}\right\}_{k=1}^{p} \cup\left\{1 / \bar{\alpha}_{k}\right\}_{k=1}^{q}$. To simplify notation, we shall use $\mathscr{R}_{n}$ to mean $\mathscr{R}_{-n, n}$. Again, to design quadrature formulas for $I_{\omega}(f)$, with positive weights, simple nodes on $\mathbb{T}$, and exact in $\mathscr{R}_{n-1}$, one should choose the nodes as the zeros of the para-orthogonal rational functions defined as

$$
Q_{n}(z, \tau)=\varphi_{n}(z)+\tau \varphi_{n}^{*}(z), \text { with } \tau \in \mathbb{T}, \text { and } \varphi_{n}^{*}(z)=B_{n}(z) \varphi_{n *}(z)
$$

where $\varphi_{n}$ is the $n$th orthogonal rational function (ORF) on $\mathbb{T}$ w.r.t. $\left(\omega,\left\{\alpha_{k}\right\}\right)$ obtained by a Gram-Schmidt orthogonalization applied to the basis $B_{0}, B_{1}, \ldots$ Also the (positive) weights can be computed in terms of the ORFs. Let us summarize this well known result as a theorem for further reference. [6,7].

Theorem 1. (Rational Szegó quadrature) Let $\left\{\varphi_{n}\right\}$ be the sequence of ORF on $\mathbb{T}$ w.r.t. $\left(\omega,\left\{\alpha_{k}\right\}\right)$ and let $Q_{n}$ be the para-ORFs (PORFs) defined by (1.2). Then

1. $Q_{n}$ has $n$ simple zeros $\left\{z_{k n}\right\}_{k=1}^{n} \subset \mathbb{T}$ 
2. The quadrature formula (1.1) satisfies $I_{n}(f)=I_{\omega}(f), \forall f \in \mathscr{R}_{n-1}$ if and only if $z_{k n}$ are the zeros of $Q_{n}$ for some $\tau$ and the (positive) weights are chosen as

$$
\lambda_{k n}=\left[\sum_{j=0}^{n-1} \frac{\left|\varphi_{j}\left(z_{k n}\right)\right|^{2}}{\left\|\varphi_{j}\right\|_{\omega}^{2}}\right]^{-1},\left\{\begin{array}{l}
\langle f, g\rangle_{\omega}=\int_{-\pi}^{\pi} f\left(\mathrm{e}^{\mathrm{i} \theta}\right) \overline{g\left(\mathrm{e}^{\mathrm{i} \theta}\right)} \omega(\theta) \mathrm{d} \theta \\
\|f\|_{\omega}^{2}=\langle f, f\rangle_{\omega} .
\end{array}\right.
$$

3. The formula $I_{n}(f)$ with positive weights and simple nodes on $\mathbb{T}$ can not be exact in $\mathscr{R}_{-(n-1), n}$ or in $\mathscr{R}_{-n, n-1}$.

Remark 1. A similar rational version of the Gauss formulas on an interval does also exist. The poles are put outside the interval of integration [21]. For the case of poles inside the support of the measure see [7, Chap. 11].

Finally we come to the rational analog on the unit circle of the Gauss-Radau and Gauss-Lobatto formulas on an interval. In the Gauss quadrature case one may fix one (Radau) or two (Lobatto) nodes in the interval of integration. Then quadrature formulas with $n+1$ or $n+2$ nodes ( $n$ nodes are free) with positive weights and all nodes simple and in the closed interval of integration and having the maximal degree of accuracy (which is $2 n-1+m$ if $m$ nodes are fixed and $n$ nodes are free) may not always exist. See [4] for a recent analysis. However, when $m=1$ or $m=2$ and the fixed nodes are placed in one or both endpoints of the interval, the corresponding quadrature formulas do exist. On the unit circle, there are no "end points" so that any node we fix can be anywhere on $\mathbb{T}$. Indeed, given $x_{1} \in \mathbb{T}$, then by trivially taking $\tau=-\varphi_{n}\left(x_{1}\right) / \varphi_{n}^{*}\left(x_{1}\right)$, the corresponding $n$-point rational Szegő quadrature formula has $x_{1}$ as a node and it is called the $n$-point Szegó-Radau formula with prescribed node at $x_{1}$. (see [5] where all $\alpha_{k}$ are zero and [9] for the general rational case.) So, the next problem, i.e. the one we want to consider in this paper, is the following. Szegó-Lobatto problem

Problem 1. (Szegó-Lobatto quadrature) Given $x_{1}, x_{2} \in \mathbb{T}, x_{1} \neq x_{2}$, and $n \geq 1$. Find $n$ distinct nodes $\left\{z_{1}, \ldots, z_{n}\right\}$, all different from $x_{1}$ and $x_{2}$ and positive weights $A_{1}, A_{2}, \lambda_{1}, \ldots, \lambda_{n}$ such that

$$
I_{\omega}(f)=\tilde{I}_{n+2}=A_{1} f\left(x_{1}\right)+A_{2} f\left(x_{2}\right)+\sum_{k=1}^{n} \lambda_{k} f\left(z_{k}\right), \quad \forall f \in \mathscr{R}_{p(n)},
$$

with $p(n)$ as large as possible.

Remark 2. Note that the largest possible $p(n)$ is $n+1$. That would happen when it was actually an $(n+2)$-point rational Szegő formula. More precisely, when $\varphi_{n}\left(x_{1}\right) / \varphi_{n}^{*}\left(x_{1}\right)=\varphi_{n}\left(x_{2}\right) / \varphi_{n}^{*}\left(x_{2}\right)$, we could simultaneously make $x_{1}$ and $x_{2}$ nodes of the quadrature formula, and then the maximal $p(n)$ is $n+1$, otherwise it will only be $n$ at most.

The problem was first tackled and solved for the polynomial case, i.e., when all $\alpha_{k}=0$ by Jagels and Reichel in [18]. See also [5] for an alternative approach and error estimates. For the rational case, see [9]. There it is shown how to solve the Radau case, and some elements are given to treat the Lobatto case as well, but its actual computation was formulated as a problem at the end of the paper [9, Problem 6.6]. In this paper, we want to deal with the computational aspects of that problem in the rational case. That is, how to compute a rational Szegó-Lobatto quadrature formula with two nodes prefixed on the unit circle, having distinct nodes with positive weights and a maximal (rational) domain of validity. So, in the next section we recall the computational aspects of generating the ORF, and their recurrence coefficients, and how these can be used to compute the nodes and weights of a rational Szegó formula. In the subsequent section, we shall show that the computation of the rational Szegó-Lobatto formula will correspond to the computation of a Szegó formula for a particular extension of the first $n$ steps in the recurrence relation with two more steps. The results given in Section 3 are actually new and simplify considerably, even in the polynomial case, the treatment that was given in [18]. Some considerations on the computational complexity are given and in the last section, we report extensively on the numerical experiments with our algorithm.

\section{Computation of the rational Szegó quadrature formulas}

We start by recalling the theorem describing the generalization of the polynomial Schur-Szegô recurrence relation since the recurrence coefficients will play a central role in the computation. We use the notation introduced in the previous section. For a proof of the following theorem see for example [7]. 
Theorem 2. Suppose that we are given a sequence $\left\{\alpha_{k}\right\} \subset \mathbb{D}$, and a positive Borel measure $\mu$ on $\mathbb{T}$. The sequence of ORF $\left\{\varphi_{k}\right\}$ with respect to $\left(\mu,\left\{\alpha_{k}\right\}\right)$ is supposed to be orthonormal with nonzero leading coefficient (with respect to the basis $\left.\left\{B_{k}\right\}\right)$, viz., $\kappa_{n}=\overline{\varphi_{n}^{*}\left(\alpha_{n}\right)} \neq 0$. Then they satisfy the recurrence relation for $n \geq 1$

$$
\begin{gathered}
{\left[\begin{array}{c}
\varphi_{n}(z) \\
\varphi_{n}^{*}(z)
\end{array}\right]=M_{n}(z)\left[\begin{array}{c}
\varphi_{n-1}(z) \\
\varphi_{n-1}^{*}(z)
\end{array}\right], \text { with }} \\
M_{n}(z)=e_{n} \frac{1-\bar{\alpha}_{n-1} z}{1-\bar{\alpha}_{n} z}\left[\begin{array}{cc}
1 & \delta_{n} \\
\bar{\delta}_{n} & 1
\end{array}\right]\left[\begin{array}{cc}
\zeta_{n-1}(z) & 0 \\
0 & 1
\end{array}\right]
\end{gathered}
$$

where

$$
\delta_{n}=\frac{\varphi_{n}\left(\alpha_{n-1}\right)}{\varphi_{n}^{*}\left(\alpha_{n-1}\right)}=-\frac{\left\langle\frac{z-\alpha_{n-1}}{1-\bar{\alpha}_{n} z} \varphi_{n-1}(z), \varphi_{k}(z)\right\rangle}{\left\langle\frac{1-\bar{\alpha}_{n-1} z}{1-\bar{\alpha}_{n} z} \varphi_{n-1}^{*}(z), \varphi_{k}(z)\right\rangle} \in \mathbb{D} \text { for any } k=0,1, \ldots, n-1
$$

and

$$
0<e_{n}=\sqrt{\frac{1-\left|\alpha_{n}\right|^{2}}{1-\left|\alpha_{n-1}\right|^{2}} \frac{1}{1-\left|\delta_{n}\right|^{2}}} .
$$

Observe that when $\alpha_{k}=0$, for $k=1,2, \ldots$, then $\delta_{n}=\rho_{n}(0)$, where $\rho_{n}$ is the $n$th monic Szegó polynomial. In that case, $\left\{\delta_{n}\right\}_{n=0}^{\infty}\left(\delta_{0}=1\right)$ is called the sequence of Verblunsky coefficients or Schur parameters or reflection coefficients.

In a few cases, the recurrence parameters $\delta_{n}$ are explicitly known, like in the case of the Lebesgue measure $\mathrm{d} \mu=\frac{\mathrm{d} \theta}{2 \pi}$ or its rational modifications [12], or for the Chebyshev weights $\omega(\theta)=(1+\cos \theta)^{a}(1-\cos \theta)^{b}$ with $a, b \in\{0,1\}$ [3] or by using a mapping from the interval to the unit circle [13].

Otherwise, if the inner products in (2.4) can be (easily) calculated, then these $\delta$ 's can be computed along with the $\varphi$ 's in a kind of Levinson-type algorithm. Yet another way is by using a kind of continued fraction expansion for the Riesz-Herglotz transform of the measure which corresponds to a Schur-Nevanlinna type interpolation algorithm [14]. The latter algorithms may however become rather unstable when the $\alpha$ 's are close to $\mathbb{T}$, even for a moderately large $n$ (see e.g. [22]).

On the other hand, if the rational moment matrix is known, i.e., when we know the matrix with entries $\left\langle B_{j}, B_{k}\right\rangle_{\mu}$, then the Levinson type algorithm to compute the expansion of the $\varphi_{k}$ with respect to the basis $\left\{B_{k}\right\}$ is equivalent with a triangular factorization of the inverse of the moment matrix and the Schur type algorithm is a matter of an LR decomposition of the moment matrix itself. If there is only a finite number of $\alpha$ 's that are repeated cyclically, the problem becomes finite dimensional and one may use a state-space formulation as in [16, 17]. All these methods compute somehow the $\varphi$ 's but not explicitly the $\delta$ 's, but when the $\varphi$ 's can be computed, then the $\delta$ 's may be obtained from (2.4).

So we assume in the rest of this paper that we know, or we are able to compute the recurrence coefficients $\delta_{k}$, $k=1,2, \ldots, n$ one way or another.

The previous theorem also has a kind of inverse which is known as a Favard theorem. See [7].

Theorem 3. (Favard theorem) Suppose that we are given a sequence $\left\{\delta_{k}\right\} \subset \mathbb{D}$, and that we generate a sequence of rational functions $\left\{\varphi_{k}\right\}$ as in (2.3) (starting e.g. with $\varphi_{0}=1$ ), then there is a positive Borel measure $\mu$ on $\mathbb{T}$ such that $\left\{\varphi_{k}\right\}$ is an ORF sequence with respect to $\left(\mu,\left\{\alpha_{k}\right\}\right)$.

In particular, suppose that $\mu$ is a positive Borel measure on $\mathbb{T}$ and that $\left\{\varphi_{k}\right\}_{k=0}^{n-1}$ are the ORFs with respect to $\left(\mu,\left\{\alpha_{k}\right\}_{k=1}^{n-1}\right)$. Choose $\delta_{n} \in \mathbb{D}$ and generate $\varphi_{n}$ as in (2.3), then there is some measure $\tilde{\mu}$ such that the sequence $\left\{\varphi_{k}\right\}_{k=0}^{n}$ is a sequence of ORFs with respect to $\left(\tilde{\mu},\left\{\alpha_{k}\right\}_{k=1}^{n}\right)$ and $\left\langle\varphi_{k}, \varphi_{l}\right\rangle_{\mu}=\left\langle\varphi_{k}, \varphi_{l}\right\rangle_{\tilde{\mu}}$ for all $k, l=0, \ldots, n-1$.

Remark 3. If in the second part of the previous theorem, we choose $\delta_{n} \in \mathbb{T}$, then the resulting $\varphi_{n}$ will not be an ORF, but it is not difficult to show that it will be para-orthogonal, which actually means that it is orthogonal to all functions in $\mathscr{L}_{n-1}$ that vanish in $\alpha_{n}$. Indeed, using the recurrence relation, it can be verified that

$$
\varphi_{n}(z)+\tau \varphi_{n}^{*}(z)=c_{n} \frac{1-\bar{\alpha}_{n-1} z}{1-\bar{\alpha}_{n} z}\left[\zeta_{n-1}(z) \varphi_{n-1}(z)+\tilde{\tau} \varphi_{n-1}^{*}(z)\right]
$$


with $c_{n}$ a nonzero constant and $\tau \in \mathbb{T}$, both depending on $\tilde{\tau}=\delta_{n} \in \mathbb{T}$. Since we are interested in its zeros to use them as nodes in an $n$-point rational Szegó quadrature formula, we could as well consider the zeros of the function

$$
X_{n}(z)=\left(z-\alpha_{n-1}\right) \varphi_{n-1}(z)+\tau\left(1-\bar{\alpha}_{n-1} z\right) \varphi_{n-1}^{*}(z), \tau \in \mathbb{T} .
$$

However, as in the case of Gauss quadrature, the computation of nodes and weights are usually not via the zeros of an orthogonal polynomial, but by an eigenvalue decomposition of the tridiagonal Jacobi matrix, also here, the weights and nodes can be computed by solving an eigenvalue problem. Therefore we need to introduce some matrices. Let $T_{\mu}$ be the multiplication operator in $L_{\mu}^{2}$, i.e., $T_{\mu} f(z)=z f(z)$. It is well known that with respect to the basis of orthogonal polynomials $\left\{\rho_{k}\right\}, T_{\mu}$ restricted to the space of polynomials has a matricial representation that is an irreducible upper Hessenberg matrix $\mathscr{H}$ [20]. It can be factorized as a product of elementary blocks

$$
\Theta_{k}=\left[\begin{array}{cc}
-\delta_{k} & \varepsilon_{k} \\
\varepsilon_{k} & \bar{\delta}_{k}
\end{array}\right] \text {, with } \delta_{k} \text { the Verblunski coefficients and } \varepsilon_{k}=\sqrt{1-\left|\delta_{k}\right|^{2}}
$$

$\Theta_{k}$ is embedded in a unit matrix at position $(k: k+1, k: k+1)$ and $\mathscr{H}$ is the product of all these elementary factors. However when, instead of the polynomial basis, we take the basis of Laurent polynomials nested according to the sequence $\left\{1, z, z^{-1}, z^{2}, z^{-2}, \ldots\right\}$, then the blocks $\Theta_{k}$ with even $k$ and with odd $k$ can be disentangled such that the matrix representation of the full $T_{\mu}$ becomes a five-diagonal matrix $\mathscr{C}$ that can be simply written as $\mathscr{C}=\mathscr{C}_{0} \mathscr{C}_{e}$ with

$$
\mathscr{C}_{o}=\left[\begin{array}{cccc}
\Theta_{1} & & & \\
& \Theta_{3} & & \\
& & \Theta_{5} & \\
& & & \ddots
\end{array}\right] \text { and } \mathscr{C}_{e}=\left[\begin{array}{cccc}
1 & & & \\
& \Theta_{2} & & \\
& & \Theta_{4} & \\
& & & \ddots
\end{array}\right]
$$

See [10].

Something similar is possible in the rational case. When the ORF basis $\left\{\varphi_{k}\right\}$, is used then the matrix representation of $T_{\mu}$ restricted to the space $\mathscr{L}_{\infty}=\cup_{n \geq 0} \mathscr{L}_{n}$ is $\mathscr{V}=\tilde{\zeta}_{\mathscr{A}}(\mathscr{H})$ where $\mathscr{H}$ is the Hessenberg matrix as before, but where the $\delta$ 's of (2.4) are used instead of the Verblunski coefficients and $\tilde{\zeta}_{\mathscr{A}}$ is a matrix Möbius transformation

$$
\tilde{\zeta}_{\mathscr{A}}(\mathscr{H})=\eta_{\mathscr{A}}^{-1}(\mathscr{H}+\mathscr{A})\left(\mathscr{I}+\mathscr{A}^{\dagger} \mathscr{H}\right)^{-1} \eta_{\mathscr{A}}^{\dagger}
$$

with $\mathscr{I}$ the identity matrix, $\eta_{\mathscr{A}}=\sqrt{\mathscr{I}-\mathscr{A}_{\mathscr{A}}^{\dagger}}, \mathscr{A}$ is the diagonal matrix with entries $\left\{\alpha_{k}\right\}$ (starting with $\alpha_{0}=0$ ) and $\mathscr{A}^{\dagger}$ is its adjoint. We could also use an orthogonal basis according to the sequence $\left\{B_{0}, B_{1}, 1 / B_{1}, B_{2}, 1 / B_{2}, \ldots\right\}$ and the matrix representation of $T_{\mu}$ becomes $\mathscr{U}=\tilde{\zeta}_{\mathscr{A}}(\mathscr{C})$ with $\mathscr{C}$ as in the polynomial case, but again replacing the Verblunski coefficients with the $\delta$ 's of (2.4). See [2, 24]. Now let us truncate all matrices to $n$ rows and $n$ columns which we indicate with a subscript $n$. Let $\delta_{1}, \ldots, \delta_{n-1}$ be the first recurrence coefficients associated with $\left(\mu,\left\{\alpha_{k}\right\}_{k=1}^{n}\right)$. Furthermore, choose $\delta_{n}=\tau \in \mathbb{T}$ and compute $\mathscr{H}_{n}, \mathscr{C}_{n}, \mathscr{C}_{\text {en }}$ and $\mathscr{C}_{\text {on }}$. Because of $\delta_{n} \in \mathbb{T}$, we call these unitary truncations. Then we have the following theorem that can be found in [2].

Theorem 4. With the unitary truncated matrices defined above with $\delta_{n} \in \mathbb{T}$, set $\mathscr{V}_{n}=\tilde{\zeta}_{\mathscr{A}_{n}}\left(\mathscr{H}_{n}\right)$ and $\mathscr{U}_{n}=\tilde{\zeta}_{\mathscr{A}_{n}}\left(\mathscr{C}_{n}\right)$. Then the following holds.

1. The zeros $z_{k}$ of the nth PORF are the eigenvalues of $\mathscr{V}_{n}$ which are also the eigenvalues of $\mathscr{U}_{n}$. If $E_{k}$ is the corresponding normalized in $\ell_{2}$ norm, then the square of its first component is the weight in the rational Szegó formula corresponding to the node $z_{k}$.

If $\varphi_{n}=p_{n} / \pi_{n}$ is generated by (2.3), then $p_{n}$ is proportional to the characteristic polynomials of $\mathscr{U}_{n}$ and of $\mathscr{V}_{n}$.

2. The zeros $z_{k}$ of the nth PORF are the generalized eigenvalues of the pencil $\left(\mathscr{C}_{\text {en }}+\mathscr{C}_{\text {on }}^{\dagger} \mathscr{A}_{n}, \mathscr{C}_{\text {on }}^{\dagger}+\mathscr{C}_{\text {en }} \mathscr{A}_{n}^{\dagger}\right)$. If the corresponding right eigenvector is $F_{n}$ and $E_{n}=\eta_{\mathscr{A}_{n}} F_{n}$, then the weight in the rational Szegóformula corresponding to $z_{k}$ is the square of the first component of the normalized vector $E_{n} /\left\|E_{n}\right\|_{2}$. 


\section{Rational Szegó-Lobatto fomulas}

Now we return to the Szegó-Lobatto problem. Suppose we are given the sequence $\left\{\alpha_{k}\right\}_{k=0}^{\infty} \subset \mathbb{D}\left(\alpha_{0}=0\right)$, and a positive Borel measure $\mu$ on $\mathbb{T}$. We shall somewhat abuse the notation $I_{\mu}(f)$ to mean $\int_{\mathbb{T}} f d \mu$ when $\mu$ is a measure instead of a weight function. Let $\left\{\delta_{k}\right\}_{k=1}^{n}$ be the first $n$ recurrence coefficients for the ORFs with respect to $\left(\mu,\left\{\alpha_{k}\right\}\right)$. Then choose $\tilde{\delta}_{n+1} \in \mathbb{D}$ and $\tilde{\delta}_{n+2} \in \mathbb{T}$, and generate

$$
\left[\begin{array}{c}
\tilde{\varphi}_{n+1}(z) \\
\tilde{\varphi}_{n+1}^{*}(z)
\end{array}\right]=\tilde{M}_{n+1}(z)\left[\begin{array}{c}
\varphi_{n}(z) \\
\varphi_{n}^{*}(z)
\end{array}\right], \quad\left[\begin{array}{c}
\tilde{\varphi}_{n+2}(z) \\
\tilde{\varphi}_{n+2}^{*}(z)
\end{array}\right]=\tilde{M}_{n+2}(z)\left[\begin{array}{c}
\tilde{\varphi}_{n+1}(z) \\
\tilde{\varphi}_{n+1}^{*}(z)
\end{array}\right]
$$

where for $k=n+1$ and $k=n+2$, the matrix $\tilde{M}_{k}(z)$ has the same form as $M_{k}(z)$ in (2.3) but with the $\delta_{k}$ replaced by $\tilde{\delta}_{k}$. By Theorem 3 we know that $\tilde{\varphi}_{n+2}$ is a PORF (depending on the chosen $\tilde{\delta}_{n+1}$ and $\tilde{\delta}_{n+2}$ ), and $\tilde{\varphi}_{n+1}$ is an ORF (depending on the chosen $\left.\tilde{\delta}_{n+1}\right)$ with respect to $\left(\tilde{\mu},\left\{\alpha_{k}\right\}\right)$ for some positive Borel measure $\tilde{\mu}$ on $\mathbb{T}$. The other functions $\left\{\tilde{\varphi}_{k}\right\}_{k=0}^{n}=\left\{\varphi_{k}\right\}_{k=0}^{n}$ are ORFs for the measure $\mu$ and hence also for $\tilde{\mu}$, since clearly $I_{\mu}(f)=I_{\tilde{\mu}}(f)$ for all $f \in \mathscr{R}_{n}$. Taking the $n+2$ zeros of $\tilde{\varphi}_{n+2}$ as the nodes of a rational Szegó quadrature formula, we know that this $(n+2)$-point formula will be exact in $\mathscr{R}_{n+1}$, and a fortiori in $\mathscr{R}_{n}$ with respect to $\tilde{\mu}$. Thus, because in $\mathscr{R}_{n}$ the integral with respect to $\mu$ and with respect to $\tilde{\mu}$ are the same, the quadrature formula will be exact in $\mathscr{R}_{n}$ with respect to $\mu$.

If we now prescribe two distinct points $x_{1}, x_{2} \in \mathbb{T}$ as the nodes that should be fixed in the Szegó-Lobatto formula, then the whole idea is to choose $\tilde{\delta}_{n+1} \in \mathbb{D}$ and $\tilde{\delta}_{n+2} \in \mathbb{T}$ such that two of the zeros of $\tilde{\varphi}_{n+2}$ coincide with $x_{1}$ and $x_{2}$. Then, because it is a rational Szegó formula (with respect to $\tilde{\mu}$ ), all its weights will be positive, the nodes will be simple, and because the formula is exact in $\mathscr{R}_{n}$ (with respect to $\mu$ ), it has the maximal degree of exactness unless $x_{1}$ and $x_{2}$ are accidentally chosen as two zeros of a PORF of degree $n+2$ for $\left(\mu,\left\{\alpha_{k}\right\}\right)$. In this particular case, the quadrature formula is an $(n+2)$-point proper rational Szegó formula that is exact in $\mathscr{R}_{n+1}$. See $[9, \S 6]$.

Define the numbers

$$
\xi_{i}=\zeta_{n+1}\left(x_{i}\right) \text { and } \tau_{i}=-\frac{\zeta_{n}\left(x_{i}\right) \varphi_{n}\left(x_{i}\right)}{\varphi_{n}^{*}\left(x_{i}\right)}, i=1,2 .
$$

Note that all these four numbers are on $\mathbb{T}$. Using the recurrence, it is directly seen that the conditions $\tilde{\varphi}_{n+2}\left(x_{i}\right)=0$ for $i=1,2$ can be written as

$$
\overline{\tilde{\delta}}_{n+1} \tilde{\delta}_{n+2}-\bar{\tau}_{i} \xi_{i} \tilde{\delta}_{n+1}-\bar{\tau}_{i} \tilde{\delta}_{n+2}+\xi_{i}=0, \quad i=1,2 .
$$

Take the equation with $i=1$ and solve for $\tilde{\delta}_{n+2}$ to get

$$
\tilde{\delta}_{n+2}=\xi_{1} \frac{\tau_{1}-\tilde{\delta}_{n+1}}{1-\tau_{1} \tilde{\tilde{\delta}}_{n+1}} .
$$

Note that $\tilde{\delta}_{n+1} \in \mathbb{D}$ (hence different from $\tau_{1} \in \mathbb{T}$ ), so that $\tilde{\delta}_{n+2}$ is well defined and will be on $\mathbb{T}$. Plug this into the equation for $i=2$ and we get

$$
\left(\bar{\tau}_{1} \xi_{1}-\bar{\tau}_{2} \xi_{2}\right)\left|\tilde{\delta}_{n+1}\right|^{2}+\bar{\tau}_{1} \bar{\tau}_{2} \tilde{\delta}_{n+1}\left(\xi_{2}-\xi_{1}\right)+\left(\xi_{2}-\xi_{1}\right) \overline{\tilde{\delta}}_{n+1}=\bar{\tau}_{1} \xi_{2}-\bar{\tau}_{2} \xi_{1} .
$$

Two cases may now be considered:

1. $\bar{\tau}_{1} \xi_{1}=\bar{\tau}_{2} \xi_{2}$. In this case, the previous relations takes the form

$$
\left[\tilde{\delta}_{n+1} \bar{\tau}_{1} \bar{\tau}_{2}+\overline{\tilde{\delta}}_{n+1}-\bar{\tau}_{1}-\bar{\tau}_{2}\right]\left(\xi_{1}-\xi_{2}\right)=0
$$

in which the trailing factor can be dropped since $\xi_{1} \neq \xi_{2}$. Note that $\tilde{\delta}_{n+1}=\tau_{1}$ and $\tilde{\delta}_{n+1}=\tau_{2}$ are two values on $\mathbb{T}$ that satisfy this equation because $\left|\tau_{i}\right|^{2}=1$. The two solutions are distinct as follows from $\xi_{1} \neq \xi_{2}$ and $\bar{\tau}_{1} \xi_{1}=\bar{\tau}_{2} \xi_{2}$. Since the equation is linear in $\tilde{\delta}_{n+1}$, and since we have two distinct solutions $\tau_{1}$ and $\tau_{2}$, all solutions are on a straight line joining $\tau_{1}$ and $\tau_{2}$. For any value of $\tilde{\delta}_{n+1}$ on the segment of that line inside the unit disk joining these two points, and for $\tilde{\delta}_{n+2}$ as in (3.7), both equations (3.6) are satisfied. 
2. $\bar{\tau}_{1} \xi_{1} \neq \bar{\tau}_{2} \xi_{2}$. Now (3.8) takes the form

$$
\left|\tilde{\delta}_{n+1}\right|^{2}-\bar{\tau}_{1} \bar{\tau}_{2} \tilde{\delta}_{n+1} c-\overline{\tilde{\delta}}_{n+1} c=\frac{\bar{\tau}_{1} \xi_{2}-\bar{\tau}_{2} \xi_{1}}{\bar{\tau}_{1} \xi_{1}-\bar{\tau}_{2} \xi_{2}}
$$

where we have set

$$
c=\frac{\xi_{1}-\xi_{2}}{\bar{\tau}_{1} \xi_{1}-\bar{\tau}_{2} \xi_{2}} \text { so that } \frac{\bar{\tau}_{2} \xi_{1}-\bar{\tau}_{1} \xi_{2}}{\bar{\tau}_{2} \xi_{2}-\bar{\tau}_{1} \xi_{1}}=1-\left(\bar{\tau}_{1}+\bar{\tau}_{2}\right) c .
$$

Note also that $\bar{c}=\bar{\tau}_{1} \bar{\tau}_{2} c$, hence $|c|^{2}+1-\left(\bar{\tau}_{1}+\bar{\tau}_{2}\right) c=\left|1-c \bar{\tau}_{2}\right|^{2}>0$. Therefore (3.9) becomes

$$
\left|\tilde{\delta}_{n+1}\right|^{2}-2 \operatorname{Re}\left(\tilde{\delta}_{n+1} \bar{c}\right)+|c|^{2}=\left|1-c \bar{\tau}_{2}\right|^{2}
$$

which is the equation of a circle $C$ with center $c$ and radius $r=\left|1-c \bar{\tau}_{2}\right|$.

(a) Suppose first that $\tau_{1} \neq \tau_{2}$, then $0<r<\infty$. We check that $\tau_{1} \in C$. Indeed, the left-hand side of (3.9) with $\tilde{\delta}_{n+1}=\tau_{1}$ becomes

$$
\left|\tau_{1}\right|^{2}-\tau_{1} \bar{c}-\bar{\tau}_{1} c=1-\bar{\tau}_{1} \tau_{1} \tau_{2} c-\bar{\tau}_{1} c=1-\left(\bar{\tau}_{1}+\bar{\tau}_{2}\right) c .
$$

Similarly, we find that also $\tau_{2} \in C$. In other words the circle $C$ intersects the unit circle $\mathbb{T}$ in the points $\tau_{1}$ and $\tau_{2}$. For $\tilde{\delta}_{n+1}$ on the arc in between lying in $\mathbb{D}$, i.e., for $\tilde{\delta}_{n+1} \in C \cap \mathbb{D}$, and $\tilde{\delta}_{n+2}$ given by (3.7), both equations of (3.6) are satisfied.

(b) If $\tau_{1}=\tau_{2}$, then $c=\tau_{1} \in \mathbb{T}$, and therefore $r=\left|1-\bar{\tau}_{1} c\right|=\left.|1-| \tau_{1}\right|^{2} \mid=0$ and $C$ reduces to a point $c=\tau_{1} \in \mathbb{T}$. Thus, there is no $\tilde{\delta}_{n+1}$ in $\mathbb{D}$ that can satisfy the equations (3.6). However, there does exist an $(n+1)$-point rational Szegó formula having the points $x_{1}$ and $x_{2}$ among its nodes. Indeed, taking $\tilde{\delta}_{n+1}=\tau_{1} \in \mathbb{T}$, the function $\tilde{\varphi}_{n+1}$ becomes a PORF for the positive Borel measure $\mu$ and we can construct a rational Szegó quadrature formula whose nodes are the zeros of $\tilde{\varphi}_{n+1}$ that is exact in $\mathscr{R}_{n}$. It remains to show that $x_{i}$, $i=1,2$ are among the nodes, i.e., the zeros of $\tilde{\varphi}_{n+1}$. Plugging $\tilde{\delta}_{n+1}=\tau_{i}$ with the expression for $\tau_{i}$ from (3.5) into the definition of $\tilde{\varphi}_{n+1}$ yields $\tilde{\varphi}_{n+1}\left(x_{i}\right)=0$. Note that $\tau_{1}=\tau_{2}$ does not mean that also $x_{1}=x_{2}$.

Remark 4. 1. Note that situation 1 can be seen as a limiting case of situation 2a because the straight line is a special case of a circle with radius $r=\infty$ and indeed $r \rightarrow \infty$, and also $c \rightarrow \infty$ as $\bar{\tau}_{1} \xi_{1} \rightarrow \bar{\tau}_{2} \xi_{2}$.

2. Similarly situation $2 \mathrm{~b}$ drops out of situation $2 \mathrm{a}$ since $r \rightarrow 0$ and $c \rightarrow \tau_{2}$ as $\tau_{1} \rightarrow \tau_{2}$. It will obviously occur when $x_{1} \rightarrow x_{2}$ but $\tau_{1} \rightarrow \tau_{2}$ also happens when $x_{1}$ and $x_{2}$ are on $\mathbb{T}$ at a distance that is exactly corresponding to the distance between two of the zeros of a PORF of degree $n+1$. Thus if we "accidentally" choose the points $x_{1}$ and $x_{2}$ to be two zeros of a PORF of degree $n+1$ with respect to $\left(\mu,\left\{\alpha_{k}\right\}\right)$. This is the same situation for PORF $\tilde{\varphi}_{n+1}$ of degree $n+1$ as we excluded at the beginning of this section for the PORF $\tilde{\varphi}_{n+2}$ of degree $n+2$.

3. If $\tau_{1} \neq \tau_{2}$ and we take $\tilde{\delta}_{n+1} \in\left\{\tau_{1}, \tau_{2}\right\}$, that is at a boundary point of the $\operatorname{arc} C \cap \mathbb{D}$, then $\tilde{\varphi}_{n+1}$ is a PORF whose zeros we can use to construct a Szegô quadrature formula, exact in $\mathscr{R}_{n}$. If $\tilde{\delta}_{n+1}=\tau_{i}$, then it is easily checked that $\tilde{\varphi}_{n+1}\left(x_{i}\right)=0$ but if $i=1$, then in general $x_{2}$ will not be among the zeros of $\tilde{\varphi}_{n+1}$ and similarly for $i=2$. We have a rational Szegó-Radau formula, which fixes only one node. The parameter $\tilde{\delta}_{n+1} \in \mathbb{T}$ can be used to shift the nodes of $\tilde{\varphi}_{n+1}$ on $\mathbb{T}$ until one of them coincides with a prescribed $x_{i}$, but since there is only one parameter, a second prescribed node can not be matched in general.

Let us summarize our results.

Theorem 5. Given a positive Borel measure on $\mathbb{T}$, and a sequence $\left\{\alpha_{k}\right\} \subset \mathbb{D}$, and two distinct points $x_{1}$ and $x_{2}$ on $\mathbb{T}$. We want to find a quadrature formula with positive weights, using $x_{1}$ and $x_{2}$ as nodes and a minimal number of other nodes, mutually distinct, such that the quadrature is exact in $\mathscr{R}_{n}$. Then we have 3 situations that are possible.

1. The points $x_{1}$ and $x_{2}$ happen to be among the zeros of a PORF of degree $n+1$ for $\left(\mu,\left\{\alpha_{k}\right\}\right)$. Then the quadrature is a rational Szegó quadrature based on that PORF and it is exact in $\mathscr{R}_{n}$.

2. The points $x_{1}$ and $x_{2}$ happen to be among the zeros of a PORF of degree $n+2$ for $\left(\mu,\left\{\alpha_{k}\right\}\right)$. Then the quadrature is a rational Szegó quadrature based on that PORF and it is exact in $\mathscr{R}_{n+1}$, hence also in $\mathscr{R}_{n}$. 
3. Otherwise there is a proper rational Szegó-Lobatto quadrature that is exact in $\mathscr{R}_{n}$. Its nodes are the zeros of the PORF $\tilde{\varphi}_{n+1}$ constructed in situation 2 a using $\tilde{\delta}_{n+2}$ defined in (3.7) and $\tilde{\delta}_{n+1} \in C \cap \mathbb{D}$, where $C$ is the circle with center $c$ defined in (3.10) and radius $r=\left|1-c \bar{\tau}_{2}\right|$. We note that $C \cap \mathbb{T}=\left\{\tau_{1}, \tau_{2}\right\}$ and that the center and the radius may be infinite, in which case the circle becomes a straight line.

This result completely answers the problem formulated in [9, Problem 6.6]. Moreover, the approach given in this section simplifies considerably the results that were formulated for the polynomial case in $[5,18]$.

Thus we have solved the Szegő-Lobatto problem as follows. First we compute the recursion coefficients $\left\{\delta_{1}, \ldots, \delta_{n}\right\}$ for $\left(\mu,\left\{\alpha_{j}\right\}\right)$. In the generic situation (case 3 of the previous theorem) we can then find a continuum of values $\tilde{\delta}_{n+1}$ on a circular arc in $\mathbb{D}$ and for each such $\tilde{\delta}_{n+1}$ a unique number $\tilde{\delta}_{n+2} \in \mathbb{T}$ such that with the sequence of parameters $\left\{\delta_{1}, \ldots, \delta_{n}, \tilde{\delta}_{n+1}, \tilde{\delta}_{n+2}\right\}$ we can generate $\tilde{\varphi}_{n+2}$ which is a PORF. All the computational techniques can be applied to obtain the nodes and weights of a Szegó quadrature formula associated with this PORF, e.g., by solving an eigenvalue problem. The resulting quadrature will be exact in $\mathscr{R}_{n}$ unless a particular choice of $x_{1}$ and $x_{2}$ accidentally results in exactness in $\mathscr{R}_{n+1}$.

\section{Computational complexity}

If the algorithm proposed in this paper is followed, then the first thing needed is to lay hands on the numerical values for $\delta_{k}$. We have mentioned several ways of computing these, some of which may be quite involving and possibly numerically unstable, evaluations of highly oscillating integrals may be needed and much will depend on how close one chooses the poles $\alpha_{k}$ to the unit circle. Even if they are "explicitly known", the expressions may be quite complex and require $O\left(n^{p}\right)$ floating point operations (flops) with $p=2$ or 3 , if not higher, to obtain the first $n$ values of $\delta_{k}$. In other cases, it may be extremely simple to compute them, like in the example of the Poisson weight (see Section 6 below) where $\delta_{1}=1$ and $\delta_{k}=0$ for $k>1$. So we no longer dwell on this in all its diversity and will have a closer look at the complexity of the algorithm when the $\delta_{k}$ have already been computed.

First we have to find $\xi_{i}$ and $\tau_{i}, i=1,2$ by formula (3.5). Using the recurrence relation, this requires $O(n)$ flops. The center $c$ and radius $r$ of the circle $C$ in (3.11) are then directly obtained. Then choose $\tilde{\delta}_{n+1}=c+r e^{\mathrm{i} \theta}$ inside $\mathbb{D}$ i.e., choose $\theta$ such that $\theta_{1}<\theta<\theta_{2}$ where $\theta_{i}=\arg \left(\tau_{i}-c\right)$. The value of $\tilde{\delta}_{n+2}$ is then given by (3.7). All this requires just a few flops.

Now that $\delta_{1}, \ldots, \delta_{n}, \tilde{\delta}_{n+1}, \tilde{\delta}_{n+2}$ are all known, the nodes and weights can be finally computed using the generalized eigenvalue problem. Suppose we choose to solve the generalized eigenvalue problem for the pencil $\left(\mathscr{C}_{\text {en }}+\right.$ $\left.\mathscr{C}_{o n}^{\dagger} \mathscr{A}_{n}, \mathscr{C}_{o n}^{\dagger}+\mathscr{C}_{e n} \mathscr{A}_{n}^{\dagger}\right)$. Computing the two tridiagonal matrices of the pencil is easy and needs ony $O(n)$ flops because $\mathscr{A}_{n}$ is diagonal and $\mathscr{C}_{e n}$ and $\mathscr{C}_{o n}$ are both block diagonal matrices with $2 \times 2$ blocks. The solution of the generalized eigenvalue problem with the standard matlab routine eig requires $O\left(n^{3}\right)$ operations. In our case it is somewhat less than for a general pencil because the matrices are in tridiagonal form. The general routine does not take this structure into account and most of the zeros are lost in the early stages of the algorithm. It might be interesting to see if it is possible to design a special purpose algorithm that does take the special structure of the pencil into account. If the (almost) Hessenberg form is used, instead of the tridiagonal form, then by using the ideas of [23] it might be possible to reduce the complexity of this step. However, since by the scaling by $\mathscr{A}_{n}$ the unitarity is lost, this might be a serious obstacle. Also the case of a symmetric weight and a mixture of real and complex conjugate pairs of poles, may reduce the work to real computations and/or half the size of the problem. However, a full investigation of the linear algebra aspects of the (generalized) eigenvalue problem is far beyond the scope of this paper and would require a separate project. For our numerical computations, given the size of the problem and the efficiency of the matlab routine eig, the computational speed was more than satisfactory. (Computing 1000 quadrature formulas each with 10 nodes took only 3 seconds on a simple desktop.) The fact that two nodes are already known in advance since they are the prefixed ones could be exploited as well, but it would probably cause more overhead than just recomputing them along with the other nodes. Their values can serve as a computational check of the accuracy of the computations. Once the eigenvalue problem has been solved, the nodes and weights are known and the rest of the computations is dominated by the number of evaluations of the integrand. This number $n+2$ will depend on the accuracy one wants to achieve and the convergence properties. This will clearly depend on how close to the unit circle $\mathbb{T}$ we will have singularities of the integrand and on how close to $\mathbb{T}$ we have chosen the $\alpha_{k}$ as will be shown in the next section. 
To give some idea of the time required, we took as an example a 150-point quadrature formula based on Chebyshev weight function and poles equal to $\alpha_{k}=1 /(k+1)$. We used matlab 7.9.0 (R2009b) under Linux Ubuntu on a Dell desktop computer with an Intel Pentium 4 processor of $3.20 \mathrm{GHz}$ and $2047 \mathrm{kB}$ cache. The average to compute the Hessenberg matrices was $1.410^{-2}$ seconds and the solution of the corresponding generalized eigenvalue probem took $3.610^{-2}$ seconds. For the construction of the five-diagonal matrices, it took $1.610^{-2}$ seconds and for the solution of the corresponding generalized eigenvalue problem 7.2 $10^{-1}$ seconds were needed. The computation of the recurrence coefficients and the evaluation of the orthogonal rational functions via the recurrence relation needed about $310^{-2}$ seconds.

\section{Error bound and convergence}

Let us now check the error of the quadrature formula in order to give some computable bound for it. Let $\tilde{I}_{n+2}(f)$ be the rational Szegó-Lobatto formula that approximates the integral $I_{\omega}(f)$ and denote the error operator as $E_{n+2}=$ $I_{\omega}-\tilde{I}_{n+2}$. We use the following notation: $\pi_{n}(z)=\prod_{k=1}^{n}\left(1-\bar{\alpha}_{k} z\right)$ and $\pi_{n}^{*}(z)=\prod_{k=1}^{n}\left(z-\alpha_{k}\right), \Gamma_{a}=\{z \in \mathbb{C}:|z|=a\}$ is a circle with radius $a$, and $\|f\|_{K}=\max \{|f(z)|: z \in K\}$. We are also assuming that $\int_{-\pi}^{\pi} \omega(\theta) \mathrm{d} \theta=1$. Then the following theorem holds.

Theorem 6. Suppose that $f$ is analytic on $\mathbb{T}$ and suppose that $\pi_{n} \pi_{n}^{*} f$ is analytic in $U \supset \mathbb{T}$. Let $r$ and $R$ be such that $0<r<1<R$ and $\Gamma_{r} \cup \Gamma_{R} \subset U$. Then

$$
\left|E_{n+2}(f)\right| \leq 2\left\|\pi_{n} \pi_{n}^{*} f\right\|_{\Gamma_{r} \cup \Gamma_{R}}\left(\frac{r^{n+1}}{1-r^{2}} g_{n}(r)+\frac{R^{1-n}}{R^{2}-1} g_{n}\left(R^{-1}\right)\right)
$$

where

$$
g_{n}(s)=\frac{1}{2 \pi} \int_{-\pi}^{\pi} \frac{\mathrm{d} \theta}{\left|\pi_{n}\left(s \mathrm{e}^{\mathrm{i} \theta}\right)\right|^{2}},|s|<1
$$

PROOF. This is a straightforward consequence of Theorem 1 in [11].

Now we see what happens if $n \rightarrow \infty$. First of all we recall the well known result: (see e.g., [25])

Lemma 1. Define $\mathscr{R}=\cup_{n=0}^{\infty} \mathscr{R}_{n}$ then $\mathscr{R}$ is dense in $\mathscr{C}(\mathbb{T})$, the space of functions continuous on $\mathbb{T}$ if and only if $\sum_{k=1}^{\infty}\left(1-\left|\alpha_{k}\right|\right)=\infty$.

Suppose $\mathscr{F}_{\omega}(\mathbb{T})=\left\{f: \mathbb{T} \rightarrow \mathbb{C}, f\right.$ bounded and $\left.f \omega \in L_{1}(\mathbb{T})\right\}$. We can then prove:

Theorem 7. If $\tilde{I}_{n+2}$ is the rational Szegö-Lobatto quadrature where for each $n$ we have chosen two points $x_{1 n}, x_{2 n} \in \mathbb{T}$, $x_{1 n} \neq x_{2 n}$, then

$$
\lim _{n \rightarrow \infty} \tilde{I}_{n+2}(f)=I_{\omega}(f), \quad \forall f \in \mathscr{F} \omega(\mathbb{T})
$$

if and only if $\sum_{k=1}^{\infty}\left(1-\left|\alpha_{k}\right|\right)=\infty$.

PROOF. Since $\tilde{I}_{n+2}(f)$ has positive weights and it is exact in $\mathscr{R}_{n}$, we can proceed in a similar way as in [6, Theorem 5 and Corollary 6].

\section{Numerical examples}

We consider the same examples that were also discussed in [2]. More details about the data used here can be found with extra information and derivations in that reference. All computations are done using matlab.

1.- For our first example we check if rational functions are integrated exactly. Take an arbitrary rational function of degree 3.

$$
f(z)=\frac{1+z}{1-z / 2}+\frac{z}{2-z}+\frac{z^{2}+3 z-z^{3}}{(z-5)(1-z / 6)(1-2 z)}
$$




\begin{tabular}{c|c|c}
$k$ & argument nodes & weights \\
\hline$z_{1}$ & $-3.098547885471644 \mathrm{e}+00$ & $5.455721876015036 \mathrm{e}+00$ \\
$\frac{x_{2}}{z_{2}}$ & $\frac{-1.884955592153877 \mathrm{e}+00}{-8.171431254732336 \mathrm{e}-01}$ & $1.028425211977852 \mathrm{e}-01$ \\
$z_{3}$ & $-1.519688644043833 \mathrm{e}-01$ & $1.484188173941068 \mathrm{e}-02$ \\
$z_{4}$ & $4.352108070744791 \mathrm{e}-01$ & $1.74879319057632 \mathrm{e}-02$ \\
$\frac{x_{1}}{z_{5}}$ & $\underline{1.256637061435917 \mathrm{e}+00}$ & $4.133547570788317 \mathrm{e}-02$ \\
$2.631490389701693 \mathrm{e}+00$ & $6.264132479906185 \mathrm{e}-01$
\end{tabular}

Table 1: The Szegô-Lobatto formula with measure $\mathrm{d} \mu(\theta)=\frac{1}{2 \pi} \frac{1-|r|^{2}}{\left|\mathrm{e}^{\mathrm{i} \theta}-r\right|^{2}}, r=-0.95$ and with $\alpha_{k}=1 /(k+1)$, $k=1, \ldots, 7$. There are two nodes prefixed: $\left\{x_{1}, x_{2}\right\}=$ $\left\{\mathrm{e}^{2 \mathrm{i} \pi / 5}, \mathrm{e}^{-3 \mathrm{i} \pi / 5}\right\}$ and 5 nodes were chosen to maximize the degree of accuracy. The table shows the arguments of the nodes, and the corresponding weights. The predefined nodes are underlined.

Note that if we take $\alpha_{k}=1 /(k+1), k \geq 1$ and $\alpha_{0}=0$, then $f \in \mathscr{R}_{-5,1} \subset \mathscr{R}_{5}$. We shall approximate $I_{\mu}\{f\}, \mu$ being the absolutely continuous measure with Poisson weight: $\mathrm{d} \mu(\theta)=\frac{1}{2 \pi} \frac{1-|r|^{2}}{\left|\mathrm{e}^{\mathrm{i} \theta}-r\right|^{2}}$ where $0 \leq r<1$. The recurrence parameters are independent of the chosen $\alpha_{k}$ and are all zero except $\delta_{1}$ which is $-r$. We have chosen as predefined nodes the arbitrary numbers $\left\{x_{1}, x_{2}\right\}=\left\{\mathrm{e}^{2 \mathrm{i} \pi / 5}, \mathrm{e}^{-3 \mathrm{i} \pi / 5}\right\} \subset \mathbb{T}$. Table 1 gives the result for the weights and nodes when we consider the rational Szegö-Lobatto formula with these two prefixed nodes, with the $\alpha_{k}$ as given above and with the remaining 5 nodes at optimal locations to guarantee a maximal domain of validity. For $\tilde{\delta}_{6} \in C \cap \mathbb{D}$ we took the point half way between $\tau_{1}$ and $\tau_{2}$. The integral is exact up to machine precision, independent of $r$, even though the 5 free nodes and all the weights do depend on $r$. If we take $\tilde{\delta}_{6} \in\left\{\tau_{1}, \tau_{2}\right\}$, then one of the weights turns out to be zero, while the integral is still exact for the rational function (6.12). We have a Szegö-Radau formula.

2.- Next we take the same weight function, but now for the integrand

$$
\frac{z}{\sin \left(z^{5}\right)+0.2}, \quad z=\mathrm{e}^{\mathrm{i} \theta}
$$

Note that it has poles that are close to the fifth roots of -0.2 . So we take $\alpha_{k}=\sqrt[5]{0.2} \mathrm{e}^{\mathrm{i} \pi(2 k-1) / 5}, k=1, \ldots, 5$ and repeat them cyclically. We choose the same prefixed nodes as before and added $n=5,6, \ldots, 150$ free nodes to be located in an optimal manner. The result is shown in figure 1. The figure top-left shows the arguments of the nodes as a function of $n$. The five dark zones, i.e., where the nodes become dense for large $n$, correspond to the part of the unit circle around the angles of the chosen $\alpha$ 's. For example $\alpha_{2}=-\sqrt[5]{0.2}$, and it seems to attract many nodes, while there are fewer nodes $\mathrm{e}^{i \theta}$ with $\theta$ near zero, which is just in between $\alpha_{0}$ and $\alpha_{4}$, which are $\sqrt[5]{0.2} \mathrm{e}^{ \pm \mathrm{i} \pi / 5}$. Note also that $x_{1}$ in between $\alpha_{0}$ and $\alpha_{1}$ is in a "light zone" and $x_{2}$ is near $\alpha_{3}$ and is in a "dark zone".

From the plot of the weights it is clear that since the weights are all positive, more and more weight will become small as $n$ increases. There are however a number of weights that persist to be dominant.

From the plot of the 10-logarithm of the relative error as a function of $n$ (bottom right), we see that there is indeed exponential convergence.

However, in this experiment, we have repeated the $\alpha_{k}$ cyclically. The five poles of the integrand are however simple, and by cyclically repeating the $\alpha_{k}$ we somehow over-emphasize the poles, which causes the nodes to be pushed away from the $\alpha$ 's. Hence the "dark" and "light" zones for the node distributions. If we however repeat the same experiment, with $\alpha_{k}, k=1,2,3,4,5$ as before, but with $\alpha_{k}=0$ for $k>5$, then the distribution of the nodes on the unit circle is much more uniform and the convergence is much better. This is illustrated in Figure 2.

3.- The Chebyshev weight function is defined as $\omega_{v}(\theta)=(1-v \cos \theta) / 2 \pi$ where $v \in\{-1,1\}$. We choose the poles by taking $\alpha_{k}=r(-1)^{k / 5}$, for $k=1,2,3,4,5$ where $r=\sqrt[5]{0.2} \approx 0.72$. All other $\alpha$ 's are equal zero. The recurrence coefficients can be computed explicitly as is explained in [2, Corollary 14]. It needs a long explanation that we do not want to repeat here. The fixed nodes are still $\left\{x_{1}, x_{2}\right\}=\left\{\mathrm{e}^{2 \mathrm{i} \pi / 5}, \mathrm{e}^{-3 \mathrm{i} \pi / 5}\right\}$ as before. The $\alpha_{k}$ are like in the previous example since we approximate now the integral

$$
\int_{-\pi}^{\pi} \frac{\mathrm{e}^{p \mathrm{i} \theta}}{\sin \mathrm{e}^{5 \mathrm{i} \theta}+0.2}(1-v \cos \theta) \frac{d \theta}{2 \pi}, \quad v \in\{ \pm 1\}, \quad p \in\{-1,0,1\} .
$$

Figure 3 gives the convergence of the relative error. Wat is shown is the 10-log of the error. The convergence is again geometric. The plots are for $v=-1$, and they are almost the same for $v=1$. On the right we have again illustrated that it is better to extend the first five $\alpha_{k}$ with zeros, instead of repeating them cyclically. 


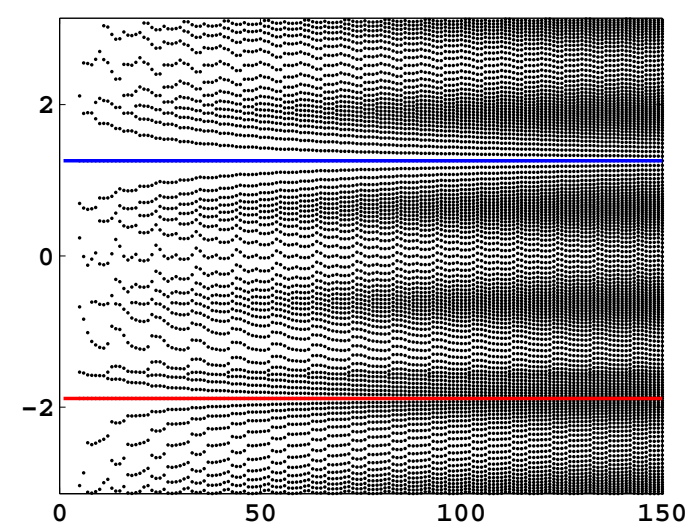

Figure 1: These figures show the rational Szegó-Lobatto formulas for the integral $\int_{-\pi}^{\pi} \frac{1-|r|^{2}}{\left|\mathrm{e}^{\mathrm{i} \theta}-r\right|^{2}} \frac{\mathrm{e}^{\mathrm{i} \theta}}{\sin \mathrm{e}^{5 \mathrm{i} \theta}+0.2}$. The value of $r=0.95 \mathrm{i}$. The two fixed nodes are $\left\{x_{1}, x_{2}\right\}=\left\{\mathrm{e}^{2 \mathrm{i} \pi / 5}, \mathrm{e}^{-3 \mathrm{i} \pi / 5}\right\}$. We took $\alpha_{k}=$ $\sqrt[5]{0.2} \mathrm{e}^{\mathrm{i}(2 k-1) \pi / 5}, k=1,2,3,4,5$ and repeat these cyclically. The quadrature formula is constructed with $n$ additional node where $n=5,6, \ldots, 150$. On top you see the argument of the nodes (left) and the $\log _{10}$ of the weights (right) as a function of $n$. The horizontal lines in the top-left figure correspond to the fixed nodes. In the figure on the right we show the $\log _{10}$ of the relative error as a function of $n$. The global geometric convergence is clear from the linear decay.
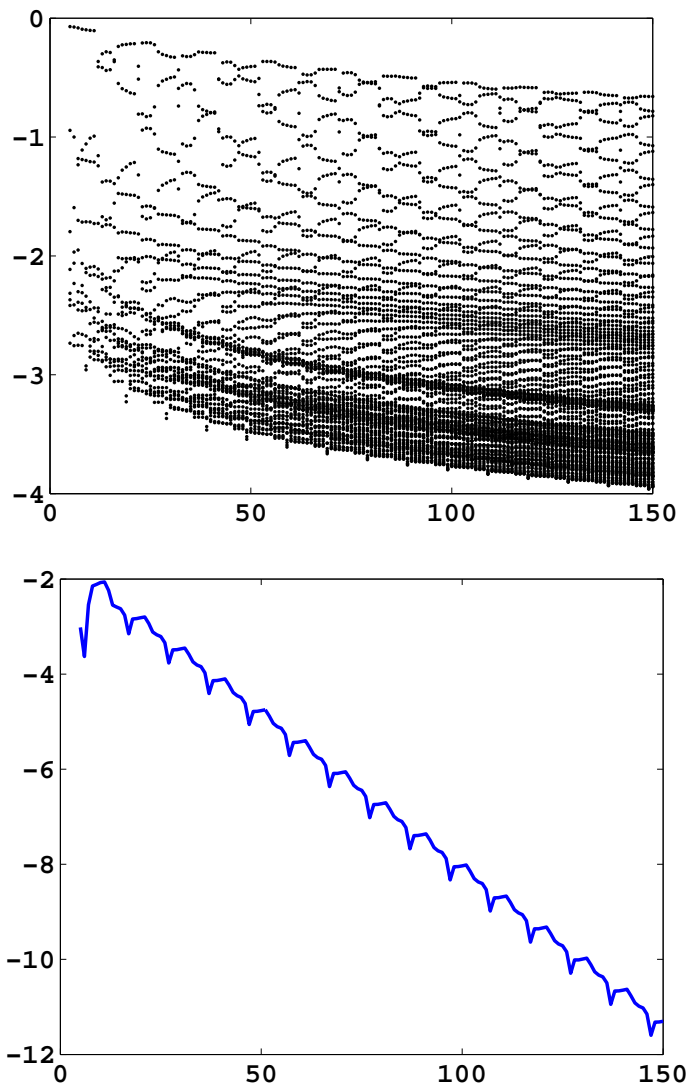

On the left plot you see 2 solid lines. The jaggy one corresponds to the case $p=0$ and $n=10,11,12, \ldots$ Note that there is a cyclic dip in the error curve every fifth value of $n$ and a somewhat deeper dip every tenth value of $n$. When we connect the error for $n=10 k, k=1,2, \ldots$, the smooth solid line results. We compute also the error for every tenth value of $n$ in the case $p=1$ and $p=-1$ which gives the dashed line and the dash-dotted line respectively.

4.- Finally, we take again the Chebyshev weights of the previous example and compute the nodes and weights. The $\alpha_{k}$ 's are equal to $r \mathrm{e}^{\mathrm{i} \pi(2 k-1) / 5}, k=1,2,3,4,5$ but now with $r=0.9$, which is closer to the circle than in the previous example. All other $\alpha_{k}$ are zero. We have computed nodes and weights of a rational Szegó-Lobatto formula with $n=5$ free nodes and the prefixed nodes equal to the complex conjugate pair $\mathrm{e}^{ \pm \mathrm{i} \pi / 5}$. Here we illustrate the behaviour of the nods and weights if we let $\tilde{\delta}_{n+1}$ loop through the circle $C$. Only part of it will be inside the open unit disk, so that only in that part we shall get a proper rational Szegó-Lobatto formula. This is illustrated in the Figures 5 and 4 . Note that for the nodes plots (left top) lines represent the map is from the argument on $C$ to the arguments on $\mathbb{T}$ so that the left and right borders match, but also the bottom and top borders do.

\section{Conclusion}

We have illustrated how rational Szegö-Lobatto formulas can be constructed. These formulas approximate an integral over the complex unit circle with positive weights, with nodes all on the unit circle and with two nodes prescribed. Moreover, a sequence of nested spaces of rational functions are associated with a given sequence of points inside the unit disk. The quadrature formulas that are constructed are exact in the largest possible space in this sequence. Of course these quadrature formulas can also be used to approximate integrals of $2 \pi$-periodic functions. We have analyzed the existence of such formulas and gave a practical computational procedure to compute the nodes and the weights of the formulas by solving an eigenvalue problem. Error bounds and a convergence theorem are provided and numerical examples illustrate the behaviour of the formulas. 


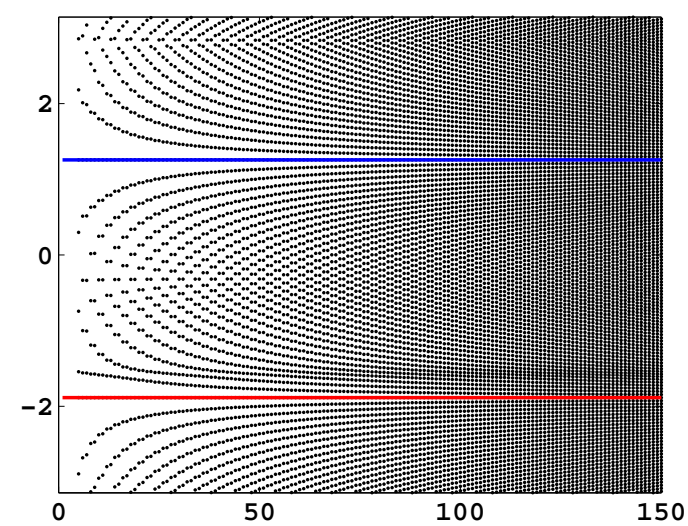

Figure 2: These figures show the rational Szego-Lobatto formulas for the integral $\int_{-\pi}^{\pi} \frac{1-|r|^{2}}{\left|\mathrm{e}^{\mathrm{i} \theta}-r\right|^{2}} \frac{\mathrm{e}^{\mathrm{i} \theta}}{\sin \mathrm{e}^{5 \mathrm{i} \theta}+0.2}$. The value of $r=0.95 \mathrm{i}$. The two fixed nodes are $\left\{x_{1}, x_{2}\right\}=\left\{\mathrm{e}^{2 \mathrm{i} \pi / 5}, \mathrm{e}^{-3 \mathrm{i} \pi / 5}\right\}$. We took $\alpha_{k}=$ $\sqrt[5]{0.2} \mathrm{e}^{\mathrm{i}(2 k-1) \pi / 5}, k=1,2,3,4$ and all other $\alpha_{k}=0$. The quadrature formula is constructed with $n$ additional node where $n=5,6, \ldots, 150$. On top you see the argument of the nodes (left) and the $\log _{10}$ of the weights (right) as a function of $n$. The horizontal lines in the top-left figure correspond to the fixed nodes. In the figure on the right we show the $\log _{10}$ of the relative error as a function of $n$. The solid line is for this experiment, the dashed line is from Figure 1 for comparison.
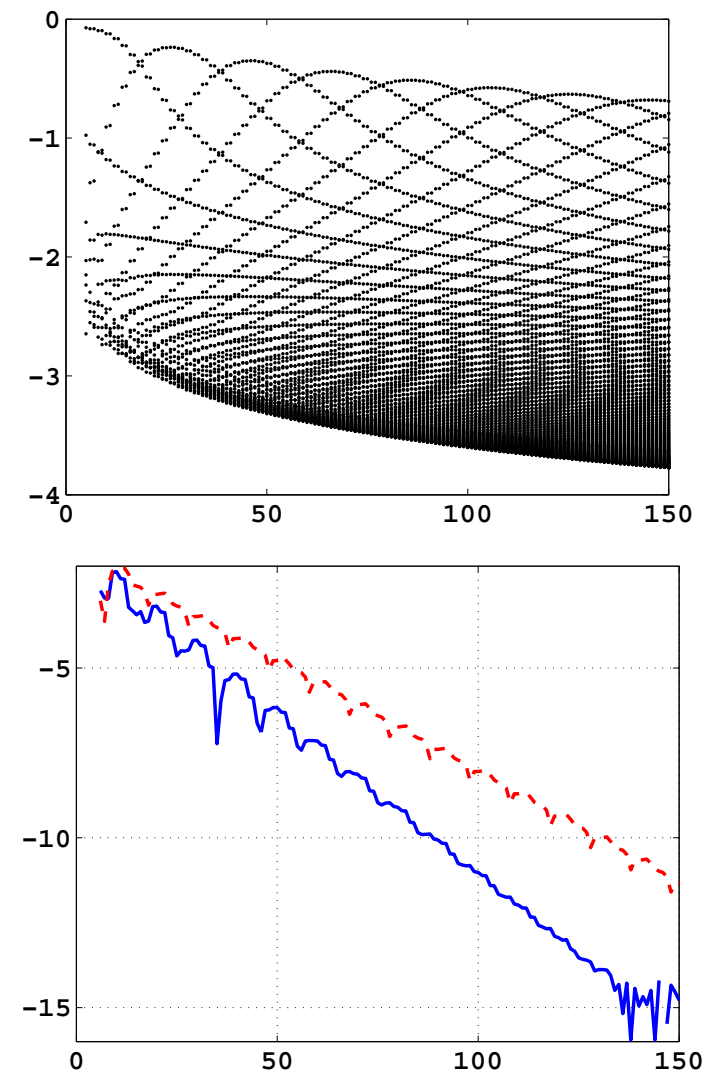

\section{References}

[1] G. Ammar, W. Gragg, L. Reichel, Constructing a unitary Hessenberg matrix from spectral data, in: G. Golub, P. Van Dooren (eds.), Numerical linear algebra, digital signal processing and parallel algorithms, vol. 70 of NATO-ASI Series, F: Computer and Systems Sciences, Springer, Berlin, 1991, pp. 385-395.

[2] A. Bultheel, M. Cantero, A matricial computation of rational quadrature formulas on the unit circle, Numer. Algorithms 52 (1) (2009) 47-68.

[3] A. Bultheel, R. Cruz-Barroso, K. Deckers, P. González-Vera, Rational Szegő quadratures associated with Chebyshev weight functions, Math. Comp. 78 (2009) 1031-1059.

[4] A. Bultheel, R. Cruz-Barroso, M. Van Barel, On Gauss-type quadrature formulas with prescribed nodes anywhere on the real line, Calcolo 47 (1) (2010) 28-41.

[5] A. Bultheel, L. Daruis, P. González-Vera, Quadrature formulas on the unit circle with prescribed nodes and maximal domain of validity, J. Comput. Appl. Math. 231 (2) (2009) 948-963.

[6] A. Bultheel, P. González-Vera, E. Hendriksen, O. Njåstad, Quadrature formulas on the unit circle based on rational functions, J. Comput. Appl. Math. 50 (1994) 159-170.

[7] A. Bultheel, P. González-Vera, E. Hendriksen, O. Njåstad, Orthogonal rational functions, vol. 5 of Cambridge Monographs on Applied and Computational Mathematics, Cambridge University Press, 1999.

[8] A. Bultheel, P. González-Vera, E. Hendriksen, O. Njåstad, Orthogonal rational functions and interpolatory product rules on the unit circle. III. Convergence of general sequences, Analysis 20 (2000) 99-120. 

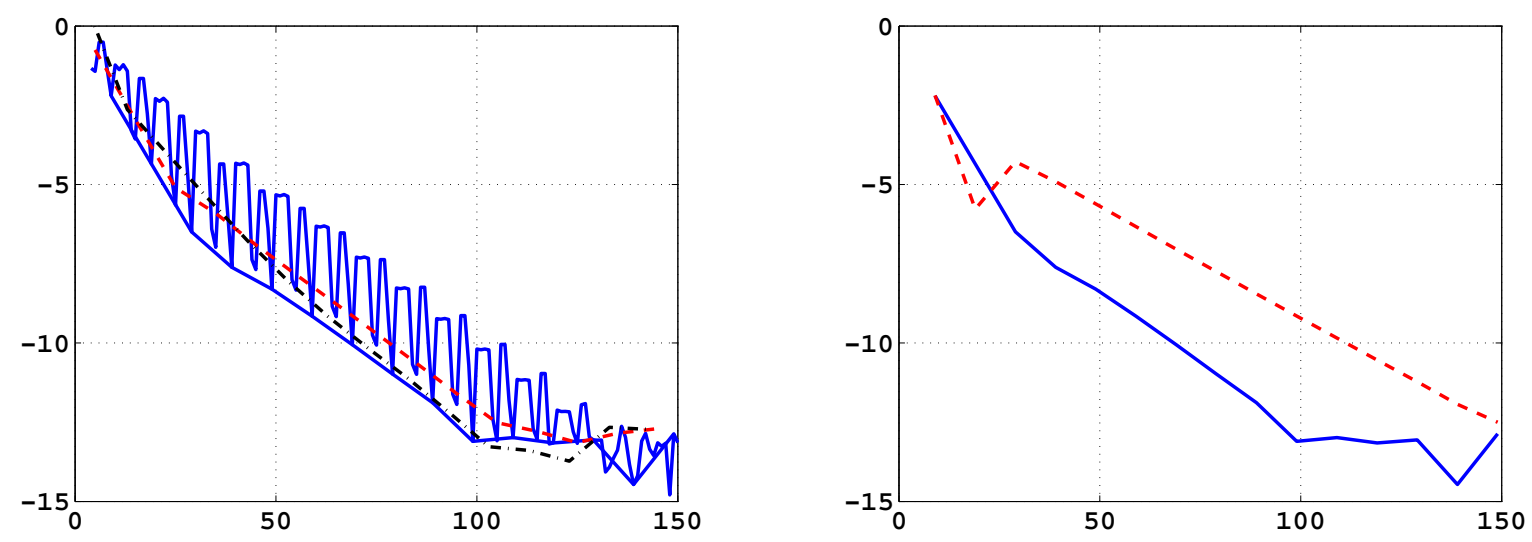

Figure 3: The example is for the Chebyshev weight function $w(\theta)=(1-v \cos \theta) / 2 \pi$. On the left, $v=1$ (for $v=-1$ the plot is very similar). The integrand is $\frac{\mathrm{e}^{p i \theta}}{\sin \mathrm{e}^{5 \mathrm{i} \theta}+0.2}$. We took $\alpha_{k}=\sqrt[5]{0.2} \mathrm{e}^{\mathrm{i}(2 k-1) \pi / 5}, k=1,2,3,4,5$ and all other $\alpha_{k}=0$. For $p=0$ (solid line), $p=1$ (dashed line), and $p=-1$ (dash-dot line). For $p=0$ we have plotted the 10-log of the relative error as a function for every $n=10, \ldots, 150$ which is the jaggy line and then connected every tenth point, which shows the smooth convergence. For $p= \pm 1$, we have only connected every tenth point. On the right $p=1, v=1$ and the solid line is the same as in the left figure. The dashed line is for the same experiment but with the first five $\alpha$ 's cyclically repeated. The same accuracy (about 13 digits) is also reached in the end but convergence is somewhat slower.
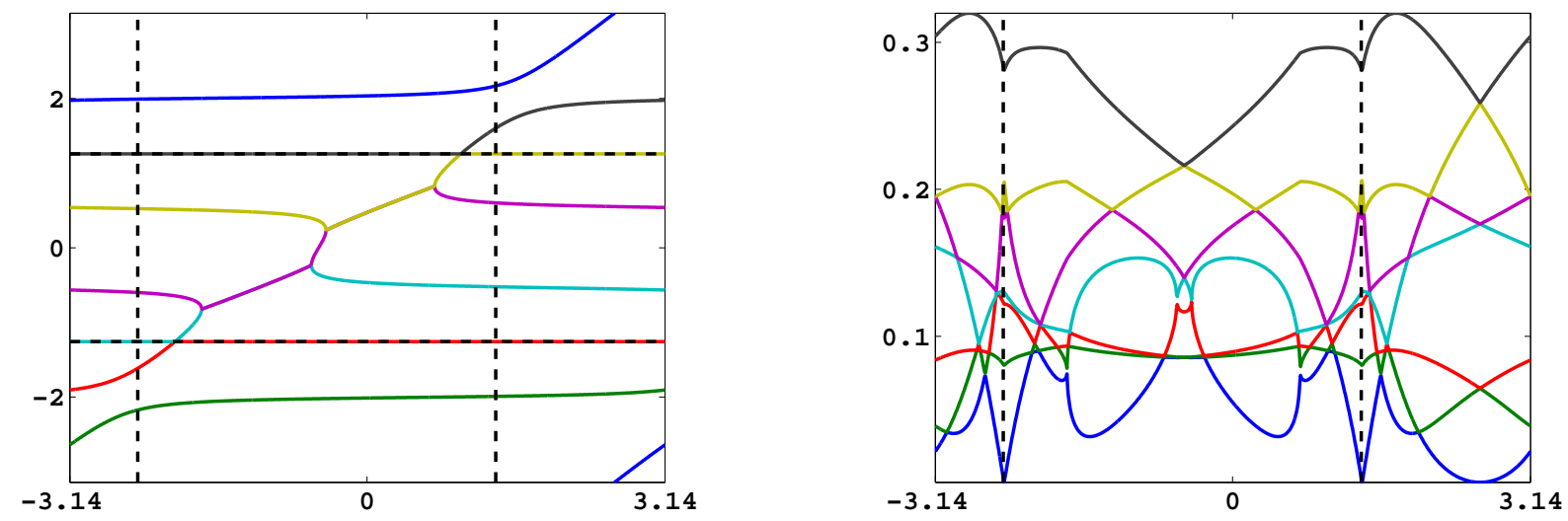

Figure 4: The example is for the Chebyshev weight function $w(\theta)=(1+\cos \theta) / 2 \pi$, the unit circle is on the right with the five $\alpha$ 's indicated by a $\times$ and cyclically repeated. The circle in solid line is the unit circle with the two prefixed nodes indicated by a *. The dashed circle is the circle $C$ with center $c$ indicated by a + . The part inside the unit disk is drawn in solid line. The two $\circ$ points are the intersection $C \cap \mathbb{T}=\left\{\tau_{1}, \tau_{2}\right\}$. On top you see the arguments of the nodes (left) and the absolute values of the weights (right) as a function of $\gamma$ where $\tilde{\delta}_{n+1}-c=\mathrm{e}^{\mathrm{i} \gamma}$ with $\gamma \in[-\pi, \pi]$, that means that $\tilde{\delta}_{n+1}$ runs counterclockwise over the circle $C$ starting on its leftmost point. It will cross $\mathbb{T}$ at the points $\tau_{1}$ and $\tau_{2}$ which are indicated in the top figures by vertical dashed lines. The straight horizontal lines in the plot of the nodes (left top) are the two prefixed nodes. The "good" interval for $\tilde{\delta}_{n+1}$, i.e., the part of $C$ inside $\mathbb{D}$, is outside the two vertical dashed lines. Since we plotted the absolute values of the weights (top right) it is seen that two of the weights have cusp points in $\tau_{1}$ and $\tau_{2}$ which indicates that they will actually become nonpositive outside the "good" interval. Note also that in the bad interval, in between the vertical lines, the nodes sometimes coincide, so that there are only 4 nodes instead of 5 besides the prefixed ones.

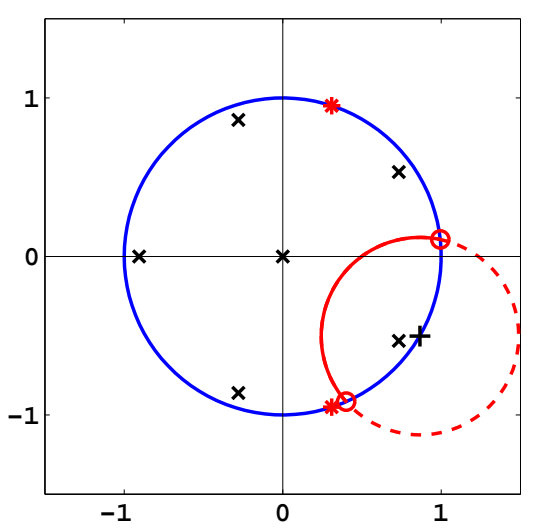




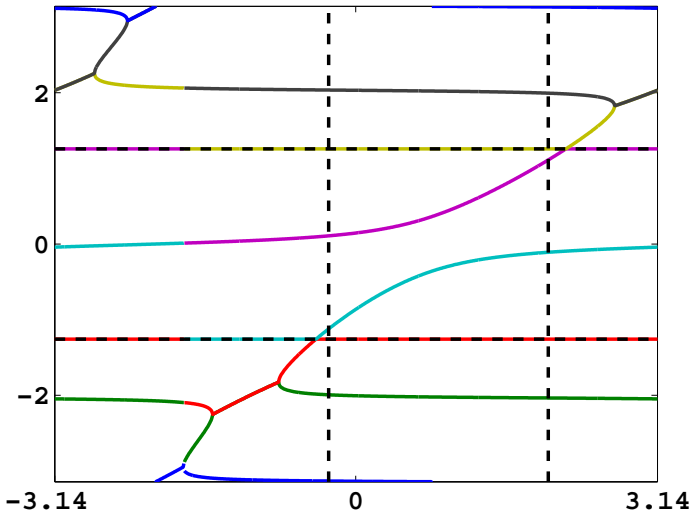

Figure 5: The example is for the Chebyshev weight function $w(\theta)=(1-\cos \theta) / 2 \pi$, the unit circle is on the right with the five $\alpha$ 's indicated by a $\times$ and cyclically repeated. The circle in solid line is the unit circle with the two prefixed nodes indicated by a $*$. The dashed circle is the circle $C$ with center $c$ indicated by a + . The part inside the unit disk is drawn in solid line. The two o points are the intersection $C \cap \mathbb{T}=\left\{\tau_{1}, \tau_{2}\right\}$. On top you see the arguments of the nodes (left) and the absolute values of the weights as a function of $\gamma$ where $\tilde{\delta}_{n+1}-c=\mathrm{e}^{\mathrm{i} \gamma}$ with $\gamma \in[-\pi, \pi]$, that means that $\tilde{\delta}_{n+1}$ runs counterclockwise over the circle $C$ starting on its leftmost point. It will cross $\mathbb{T}$ in the points $\tau_{1}$ and $\tau_{2}$ which are indicated in the top figures by vertical dashed lines. The straight horizontal lines in the plot of the nodes (left top) are the two prefixed nodes. The "good" interval for $\tilde{\delta}_{n+1}$ is in between the two vertical dashed lines. Since we plotted the absolute values of the weights (top right) it is seen that some weights have a cusp points in $\tau_{1}$ and/or $\tau_{2}$ which indicates that they will probably become nonpositive .
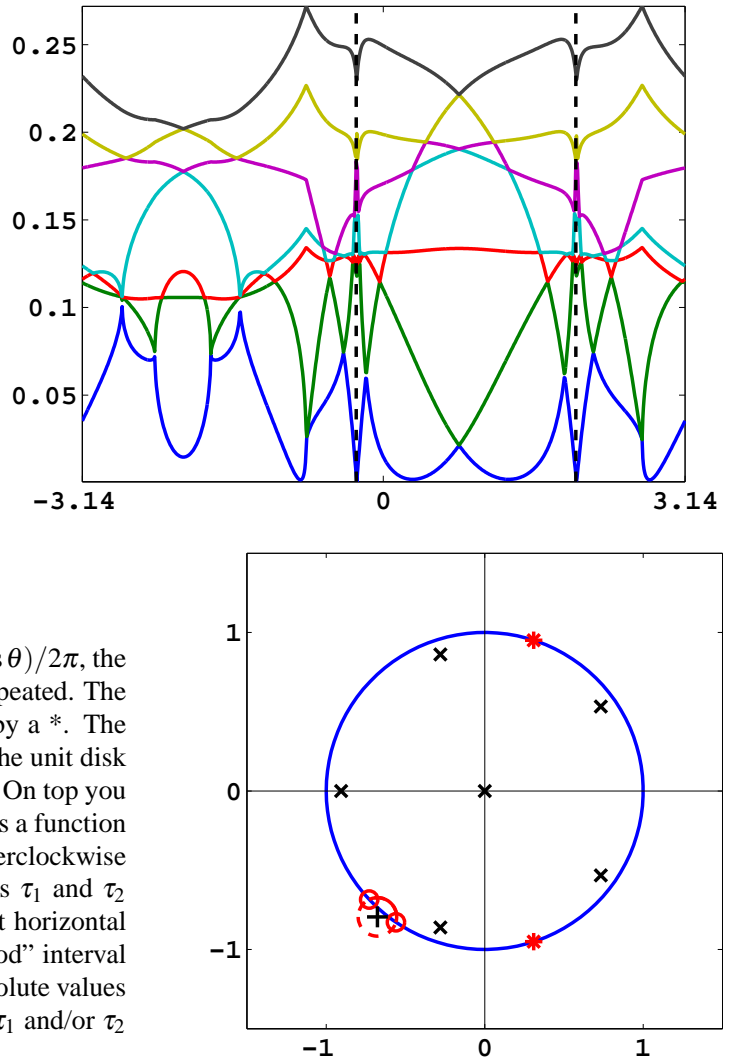

[9] A. Bultheel, P. González-Vera, E. Hendriksen, O. Njåstad, Rational quadrature formulas on the unit circle with prescribed nodes and maximal domain of validity, IMA J. Numer. Anal.Published on line August 21, 2009.

[10] M. Cantero, L. Moral, L. Velázquez, Five-diagonal matrices and zeros of orthogonal polynomials on the unit circle, Linear Algebra Appl. 362 (2003) 29-56.

[11] B. de la Calle Ysern, P. González-Vera, Rational quadrature formulas on the unit circle with arbitrary poles, Numer. Math. 107 (4) (2007) 559-587.

[12] K. Deckers, A. Bultheel, Orthogonal rational functions and rational modifications of a measure on the unit circle, J. Approx. Theory 157 (2009) 1-18, published online 13 October 2008.

[13] K. Deckers, J. Van Deun, A. Bultheel, An extended relation between orthogonal rational functions on the unit circle and the interval [-1,1], J. Math. Anal. Appl. 334 (2) (2007) 1260-1275.

[14] P. Dewilde, H. Dym, Schur recursions, error formulas, and convergence of rational estimators for stationary stochastic sequences, IEEE Trans. Inf. Th. IT-27 (1981) 446-461.

[15] W. Gragg, Positive definite Toeplitz matrices, the Arnoldi process for isometric operators and Gaussian quadrature on the unit circle, in: E. Nikolaev (ed.), Numerical methods in linear algebra, Moscow university press, 1982, pp. 16-32, (Russian, English translation in J. Comput. Appl. Math., 46 (1-2) (1993) 183-198.).

[16] P. Heuberger, T. de Hoog, P. Van den Hof, B. Wahlberg, Ortohonormal basis functions in time and frequency domain: Hambo transform theory, SIAM J. Control Optim. 42 (4) (2003) 1347-1373.

[17] P. Heuberger, P. Van den Hof, B. Wahlberg (eds.), Modelling and Identification with Rational Orthogonal Basis Functions, Springer Verlag, 2005. 
[18] C. Jagels, L. Reichel, Szegő-Lobatto quadrature rules, J. Comput. Appl. Math. 200 (1) (2007) 116-126.

[19] W. Jones, O. Njåstad, W. Thron, Moment theory, orthogonal polynomials, quadrature and continued fractions associated with the unit circle, Bull. London Math. Soc. 21 (1989) 113-152.

[20] B. Simon, Orthogonal polynomials on the unit circle, vol. 54 of Colloquium Publications, AMS, 2005.

[21] J. Van Deun, A. Bultheel, Orthogonal rational functions and quadrature on an interval, J. Comput. Appl. Math. $153(1-2)(2003) 487-495$.

[22] J. Van Deun, A. Bultheel, An interpolation algorithm for orthogonal rational functions, J. Comput. Appl. Math. 164-165 (2004) 749-762.

[23] R. Vandebril, M. Van Barel, N. Mastronardi, Matrix Computations and Semiseparable Matrices. Volume 2: Eigenvalue and Singular Value Methods, The Johns Hopkins University Press, 2008.

[24] L. Velázquez, Spectral methods for orthogonal rational functions, J. Funct. Anal. 254 (4) (2008) 954-986.

[25] J. Walsh, Interpolation and approximation, vol. 20 of Amer. Math. Soc. Colloq. Publ., 3rd ed., Amer. Math. Soc., Providence, Rhode Island, 1960, first edition 1935. 